\title{
Research on Application of Mobile Agent Based on Immune Algorithms in Ad Hoc Network
}

\author{
Qinggong Ma, Bo Yang \\ Changzhou University Huaide College, Taizhou, China \\ Email: hd.xxzx@cczu.edu.cn
}

Received 14 September 2015; accepted 26 October 2015; published 29 October 2015

Copyright (C) 2015 by authors and Scientific Research Publishing Inc.

This work is licensed under the Creative Commons Attribution International License (CC BY). http://creativecommons.org/licenses/by/4.0/

cC) (7) Open Access

\begin{abstract}
Ad Hoc network is a kind of multi hop, self-organizing wireless network without center. Each node in network can be used as host as well as router and it can form any network topology through wireless connections. Because of characteristics of itself, many new service items and application fields appear; meanwhile, it is also faced with many new security threats. Using immune agent can perceive the change of network node and make corresponding decision behavior, find the misbehavior nodes in the network as soon as possible and reduce the network attack and improve the immune competence of network.
\end{abstract}

\section{Keywords}

Immune Network, Mobile Agent, Ad Hoc Network Security

\section{Introduction}

Mobile Ad Hoc network [1] is a collection of multiple mobile nodes that can communicate with each other without fixed infrastructure and network topology can arbitrarily change. As a kind of special wireless network, nodes in network generally consist of mobile terminal. Because Ad Hoc network has characteristics of open media, dynamic topology, lack of center authentication mechanism, and limited node ability, compared with traditional network, it has more network security problems. Security problems have become choke point to hinder the development of Ad Hoc network. Attacks in Ad Hoc network can be divided into "active attack" and "passive attack". Active attack can be detected easily but passive attack has high concealment and it is not easy to be found [2]. While mobile agent based on immune system can well monitor each node in the network, timely find its misconduct and make use of the immune ability of the network to restore the health of network nodes or 
eliminate it out of the entire network. This article will focus on how to apply immune algorithms and mobile agent to improve network security performance in Ad Hoc network.

\section{Immunology Principle}

\subsection{Immune System}

Immune system is a highly collateral distributed, adaptive information processing learning system [3]. The system can recognize self and non-self materials, eliminate and defend foreign invasive viral substances or molecules. In order to recognize self and non-self materials, in biological systems, it usually has $\mathrm{T}$ cells that specially detect antigen and B cells that produce antibody. There are two types of T cells. One kind is the helper T cell Th that is in the majority to be responsible for promoting B cells to secrete antibodies and strengthen the immune function of $\mathrm{T}$ cells and macrophage; another kind is suppressor $\mathrm{T}$ cell Ts, which activates under the action of cytokines to form effector cell and eliminate bad cells.

The immune response can be divided into three phages: immune recognition phase, antibody production stage, antigen elimination stage.

1) Immune recognition phase: when external antigen enters in the system, at first, the $T$ cell does immunological recognition. If it recognized similar antigen and retain the information before, the memory part of $\mathrm{T}$ cell will combine with the antigen to realize immunological recognition;

2) Antibody production stage: if $T$ cell has recognized the antigen, $T$ cell will be activated and stimulate $B$ cell to secrete large amounts of antibodies;

3) Antigen elimination stage: antibodies produced by B cell combine with antigen, which destroy the activity of antigen and it excretes the antigen out of the body. The antigen disappears, and the immune system returns to normal.

\subsection{Immune Algorithms}

Immune algorithm is the learning algorithm based on the immune system [4], and there are four basic elements, antigen recognition, antibody formation, immune selection and immune memory respectively. In the algorithm, regard the objective function of the optimal solution as the antigen, including problems to be solved and various constraint conditions. Solve the fitness function $f\left(x_{i}\right)$ of $x_{i}$ and regard it as antigen, search the direction and the distribution of solution group according to concentration adaptive modulation of antibody. The representation of immune selection is $\frac{\sum_{j=1}^{N}\left|f\left(x_{i}\right)-f\left(x_{j}\right)\right|}{\sum_{i=1}^{N} \sum_{j=1}^{N}\left|f\left(x_{i}\right)-f\left(x_{j}\right)\right|}$. Immune memory refers to reserving the solving result of a specific problem and characteristics and parameters of the problem as initial solutions to solve the similar problems next time so as to accelerate the solving speed and effect.

The process to realize immune algorithms is as follows:

1) Initialization, randomly generate initial B cell mass $P_{0}$.

2) Calculate the fitness of each $B$ cell to generate antibodies $f\left(x_{k}\right)$.

3) Save B cell with the optimal antibody as immune memory cell $x_{k}^{\max }$.

4) Calculate the concentration of antibody and generate B cell mass $P_{k}$ according to the probability of immune selection.

5) $P_{k}$ forms the next generation $P_{k+1}$ of B cell through clone and differentiation.

6) If immune memory cell hasn’t completely recognized antigen, return to step 2).

\section{The Working Mechanism of Mobile Agent}

Agent technology derives from artificial intelligence domain [5], can simulate human behavior and relationships and it is a procedure that can run independently and provide specific service. It can sense the environment change, make judgment and reasoning for changes and form the decision to control corresponding behavior and complete a task. Compared with traditional distributed computing model, mobile agent technology has many advantages, such as save network bandwidth, support off-line calculating, unrelated to platform and balance 
computation load, etc. In the network environment, under the self-control, the mobile agent can move from one computer to another computer, hang up at any point, and it continues execution from the hanging point when moving to a new machine. Under normal circumstances, mobile agent system (MAS) includes mobile agent MA and mobile agent platform MAE. Mobile agent platform is responsible for establishing safe and correct operation environment for mobile agent, providing basic services and forming restriction mechanism, fault-tolerance strategy and security control for MA. The mobility of mobile agent and the solving ability of problems depend largely on the services provided by mobile agent platform.

\section{The Application of Immune Agents in Ad Hoc Network}

Because the Ad Hoc network topology is changeful, the establishment of a connection mode completely depends on the location of each node and coverage area of their transceiver. Each node in the network is equivalent to a router, realizing the retransmission of information packet and participating in the discovery and maintenance of route. Ad Hoc network brings convenience to people and is faced with serious security threat. Attacks from network can be divided into passive attacks and active attacks. Under passive attack mode, attackers don't interfere with the communication of network nodes and their purpose is to obtain sensitive information in the network; active attack refers to that the attackers interfere with or destroy the communication of network nodes and cause that the network nodes cannot work normally. Flooding DDOS attack is a typical attack type. In order to early find misbehavior nodes in network and maintain normal communication of the system, this article combines immune algorithm with mobile agent and applies them to Ad Hoc network, makes network node can dynamically monitor change situation of other nodes in network, timely remove or restrict the behavior of misbehavior nodes and realize autonomous management of network nodes under the circumstances of no one involved.

\subsection{Working Model of Immune Agent}

It is easy to find the corresponding relation between immune system and Ad Hoc network through analyzing the division of labor of lymphocyte, T cell, B cell and the working mechanisms of mobile agent in immune system. The security architecture of Ad Hoc network is equivalent to the entire immune system; misbehavior nodes in Ad Hoc network are equivalent to the antigens in immune system; normal nodes in Ad Hoc network are equivalent to healthy cells in immune system; mobile agent in Ad Hoc network is equivalent to lymphocyte in immune system and responsible for transporting other agents with specific function. In view of the function of $\mathrm{T}$ cell and B cell, three types of agents have been set in the system, and they are monitoring agent, coordinating agent and blocking agent respectively. Monitoring agent is equivalent to $\mathrm{T}$ cell in immune system, distributes in all the nodes of the whole system, and it is responsible for collecting behavior information of neighbor nodes and regularly reports it to coordinating agent; coordinating agent distributes in different areas of the network and it is equivalent to B cell in immune system and responsible for arranging the information of nodes in the charge of it in the network and storing it into information memory bank. When coordinating agent receives a new report, it will compare the report with the information in memory bank and strategy library. If it is normal information, the agent will ignore it; if it isn't normal behavior information (if a node receives routing information and doesn't transpond), judge the time difference of bad report at the same node in last time. If the interval is nearer, then accumulate. Otherwise, remove the bad report number of this node. If it exceeds the threshold value, activate the blocking agent. Blocking agent is equivalent to antibody in immune system and can clone itself. Its task is to eliminate the misbehavior nodes [6]. Coordinating agent needs certain tolerance and can tolerate a small amount of bad behavior of individual nodes (some bad behaviors may be caused by network interference).

The safety verification of antibody moving from point $h_{0}$ to $h_{i+1}$ is described as follows:

$$
\begin{aligned}
& O_{i}=E_{0}\left(H_{i}, S_{i}\left(o_{i}\right), \text { Uid }\right), h_{i}, 0 \leq i \leq n \\
& h_{0}=h\left(r_{0}, H_{i}\right) \\
& h_{i}=h\left(O_{i-1}, H_{i+1}\right), 1 \leq i \leq n \\
& H_{i} \rightarrow H_{i+1}:\left\{O_{k} \mid 0 \leq k \leq i\right\}
\end{aligned}
$$




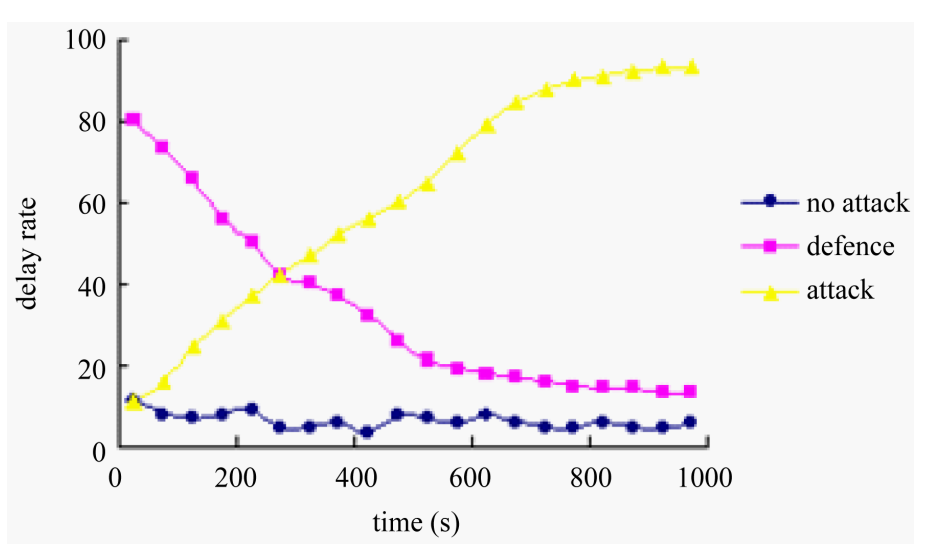

Figure 1. Results contrast figure.

When the antibody begins to roam, $H_{0}$ produces a random number $r_{0}$ and a $o_{0}$, and then calculate $h_{0}$ and $O_{0}$, send $O_{0}$ to $H_{i}$. When the antibody reaches $H_{i+1}, H_{i+1}$ checks the chain relationship $h_{k}, 1 \leq k \leq i$.

When the antibody moves to the position near the goal node, it clones, mutates and compresses [7] and produces a large number of antibodies that can kill the "virus". These antibodies will isolate the communication between misbehavior nodes and other nodes in network and achieve the purpose of eliminating harmful nodes. When misbehavior nodes disappear, blocking agent will vanish by itself.

The mathematical description of antibody clone is as follows:

For binary coding, antibody $a \in B^{l}$. Among them, $B^{l}=\{0,1\}^{l}$ represents the collection formed by all the binary strings with length of $l$. Antibody cluster $A=\left\{a_{1}, a_{2}, \cdots, a_{n}\right\}$ is $n$ tuple of antibody $a$.

Clone operator is defined as follows: $\Theta(A)=\left\{\Theta\left(a_{1}\right), \Theta\left(a_{2}\right), \cdots, \Theta\left(a_{n}\right)\right\}$. Among it, $\Theta\left(a_{i}\right)=I_{i} \times a_{i},(i=1,2, \cdots, n) . I_{i}$ is $q_{i}$ dimensional row vector $q_{i}=g\left(N_{c}, f\left(a_{i}\right)\right)$. Usually take $q_{i}=\operatorname{Int}\left[N_{c} \times \frac{f\left(a_{i}\right)}{\sum_{j=1}^{n} f\left(a_{j}\right)}\right], i=1,2, \cdots, n$. Among it, $N_{c}>n$ is concerned set value of clone size and $\operatorname{Int}(*)$ is top integral function. After the clone operation, it generates intermediate population $A^{\prime}=\left\{A, A_{1}^{\prime}, A_{2}^{\prime}, \cdots, A_{n}^{\prime}\right\}$ and $A_{i}^{\prime}=\left\{a_{i 1}, a_{i 2}, \cdots, a_{i q-1}\right\}$.

The mathematical description of the variation is as follows:

$$
p\left(a_{i} \rightarrow a_{i}^{\prime}\right)=\left\{\begin{array}{lc}
0 & a_{i} \in A \\
p_{m}^{H\left(a_{i}, a^{\prime}\right)}\left(1-p_{m}\right)^{l-H\left(a_{i}, a^{\prime}\right)} & a_{i} \in A_{j}^{\prime}
\end{array}, H\right. \text { is Hamming distance. After variation, it generates in- }
$$

termediate population $A^{\prime \prime}=\left\{A, A_{1}^{\prime \prime}, A_{2}^{\prime \prime}, \cdots, A_{n}^{\prime \prime}\right\}$ and $A_{i}^{\prime \prime}=\left\{a_{i 1}^{\prime}, a_{i 2}^{\prime}, \cdots, a_{i, q-1}^{\prime}\right\}$.

The main effect of compression choice is to choose $n$ antibodies with the highest affinity in intermediate population $A^{\prime \prime}$ to form a new generation of antibody group $A(t+1)$.

Evaluate the immune system in the form of weighted sum, and the evaluation function is

$S l=M a t+K_{1} * \operatorname{Sim}-K_{2} * \operatorname{Sup}$. In this formula, $K_{1}$ and $K_{2}$ are weighted system of stimulating effect and inhabitant activity.

\subsection{Result Analysis}

It is can be seen from Figure 1 that when there is no attack in network, the message delay maintains at a relatively low level and the network communication is normal shown in the figure as "•"; when the network appears attack, the delay rate of message will rise rapidly. With the extension of time, network performance becomes worse and worse and the worst result is network paralysis, shown as " $\mathbf{\Lambda}$ "; after applying the method of immune agent in network, the number of message delay of the system will reduce sharply, shown as "ø". Although it is higher than the message delay rate when there is no attack, it is still in a lower level and acceptable. 


\section{References}

[1] Zheng, S.R., Wang, H.T. and Zhao, Z.F. (2005) Ad Hoc Network Technology. Posts and Telecom Press, Beijing.

[2] Cohen, F. (1999) Simulating Cyber Attacks, Defenses, and Consequences [EB/OL]. http://all.net/journal/ntb/simulate/simulate.html

[3] Steve, C. and Uwe, A. (2002) A Recommender System Based on the Immune Network. Proceedings CEC 2002, Honolulu, 807-813.

[4] Wang, L. (2001) Intelligent Optimization Algorithms with Applications. Tsinghua University Press, Beijing.

[5] Magic, G. (2012) Telescript/Odyssey [EB/OL]. http://www.genmagic.com/agents

[6] Agent Transport Protocol Specification. IBM Aglet Work Bench [EB/OL]. http://www.trl.ibm.com.jp/aglets

[7] Zhou, G.Y. (2000) Principles of Immunology. Shanghai Scientific and Technological Literature Press, Shanghai. 